\title{
Lyon $\left(5^{\mathrm{e}}\right)$ - 23-29 chemin de Montauban, Le Clos de la Solitude
}

Sondage (2013)

Benjamin Clément

\section{(2) OpenEdition \\ Journals}

Édition électronique

URL : https://journals.openedition.org/adlfi/15566

ISSN : 2114-0502

\section{Éditeur}

Ministère de la Culture

\section{Référence électronique}

Benjamin Clément, « Lyon (5e) - 23-29 chemin de Montauban, Le Clos de la Solitude » [notice

archéologique], ADLFI. Archéologie de la France - Informations [En ligne], Auvergne-Rhône-Alpes, mis en ligne le 28 août 2015, consulté le 28 juillet 2021. URL : http://journals.openedition.org/adlfi/15566

Ce document a été généré automatiquement le 28 juillet 2021.

(c) ministère de la Culture et de la Communication, CNRS 


\section{Lyon $\left(5^{\mathrm{e}}\right)-23-29$ chemin de Montauban, Le Clos de la Solitude}

Sondage (2013)

\section{Benjamin Clément}

\section{NOTE DE L'ÉDITEUR}

Organisme porteur de l'opération : Université Lumière Lyon II

1 Les sondages archéologiques effectués sur le clos de la Solitude ont été proposés dans le cadre d'une thèse de doctorat portant sur l'architecture domestique à Lugudunum et dirigée par M. Poux et A. Desbat, à l'Université Lyon 2. Ils font suite à la reprise du mobilier issu des fouilles anciennes menées sur le site par le personnel de l'école. Ces sondages, au nombre de trois, ont porté sur la moitié sud de la propriété et avaient pour objectif d'étudier de manière exhaustive les matériaux et les modes de construction utilisés pour les bâtiments antiques en partie dégagés dans les années 1960 (fig. 1). Ces sondages ont également permis de mieux cerner l'évolution chronologique et fonctionnelle des îlots méridionaux du clos de la Solitude, dont seuls quelques relevés sommaires ont été dressés dans les années 1960 (Gruyer 1973). 
Fig. 1 -Localisation de sondages

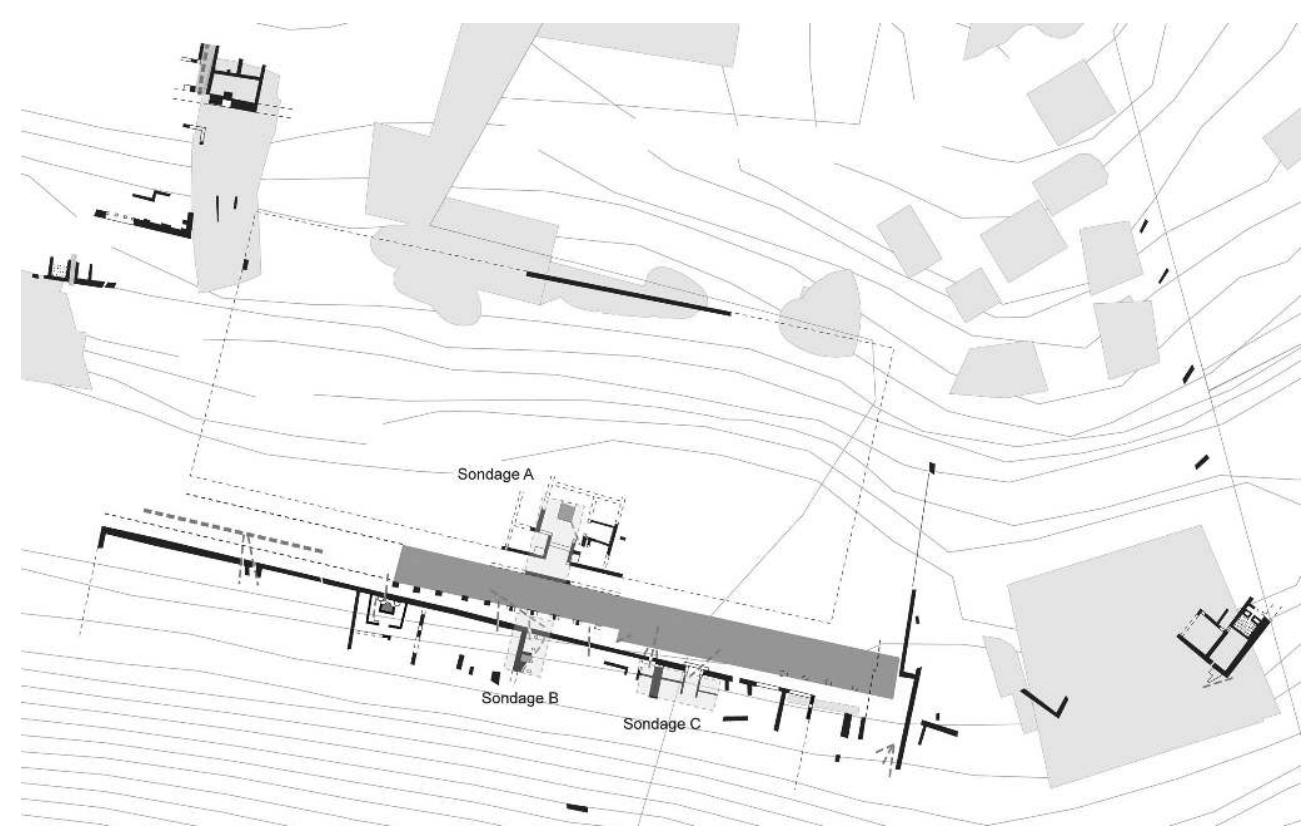

DAO : B. Clément (université Lyon II).

Le sondage A, d'une superficie de $50 \mathrm{~m}^{2}$, a été implanté dans une jardinière disposée au centre du terrain de sport. Il a permis de dégager à nouveau la maison au Dauphin, en partie observée dans les années 1963/1964. Des niveaux de la fin de l'âge du Fer (fosse/ fossé, espace de circulation) ont été atteints sur une fenêtre réduite dans la partie nord du sondage. Daté des années 150/90 av. J.-C., il constitue le premier état d'occupation $\mathrm{du}$ site dont l'organisation nous échappe. La maison au Dauphin est construite dans les années $20 \mathrm{av}$. J.-C. et oblitère les niveaux d'époque laténienne. Elle présente dès cette époque un vaste vestibule de 3,60 $\mathrm{m}$ de large donnant au sud sur un portique longeant la rue et au nord sur un atrium sans doute doté d'un impluvium. À partir du milieu du $\mathrm{I}^{\mathrm{er}} \mathrm{s}$. apr. J.-C., l'atrium de la domus est entièrement remanié avec la réfection de son décor (sol en opus crustatum avec emblema et nouvelle peinture murale) et l'installation d'un nouveau bassin en matériaux précieux (calcaire ou marbre ?) dont le négatif des orthostates a pu être observé. Il semble que le vestibule soit condamné à cette époque. La domus est sans doute abandonnée suite à un incendie violent qui détruit le quartier dans le premier quart du $\mathrm{II}^{\mathrm{e}} \mathrm{s}$. apr. J.-C. L'arasement des vestiges dans ce secteur, en raison des travaux de construction de la piste de sport, n'a pas permis d'appréhender des niveaux plus récents.

Dans l'axe du premier sondage, le sondage B a été implanté dans le talus de la piste de sport en gore (superficie de $60 \mathrm{~m}^{2}$ ). En partie dégagé dans les années 1960, ce sondage a permis de reconnaître la façade de la parcelle aux «lits de briques » ainsi que son portique. Sur d'importants niveaux de remblai/colluvion, deux boutiques caractérisées par deux antes maçonnés, encadrant un vestibule, sont construites autour des années 20/15 av. J.-C. Elles fonctionnent avec un portique marqué par des bases maçonnées délimitant un trottoir et la rue. Ces boutiques subissent de nombreuses réfections (sols, réseau hydraulique et maçonneries) jusqu'au début de l'époque flavienne où deux boutiques sont aménagées. La boutique orientale, divisée en au moins deux nefs par une série de piliers, est dotée d'une canalisation et d'un bassin en bois. La boutique occidentale, partiellement fouillée, correspond à un atelier de 
tabletterie. Suite à un incendie violent qui survient dans les années 120/140 apr. J.-C., ces boutiques sont entièrement détruites et leurs niveaux de démolition ont été conservés en place. Ils permettent de révéler l'organisation de l'étage qui les surplombait. Ce dernier est dévolu à un habitat assez luxueux (sols en opus crustatum) dont la fouille a permis de découvrir trois coffres en bois avec leurs contenus (vaisselle en bronze et en verre, harnachement, hipposandale...). Suite à cet incendie, on assiste à un rehaussement du quartier d'environ 2,50 $\mathrm{m}$ avant la construction de la maison aux Lits de Briques, au milieu du $\mathrm{II}^{\mathrm{e}} \mathrm{s}$. apr. J.-C. Cette dernière sera reconstruite à la fin du $\mathrm{II}^{\mathrm{e}}$ ou au début du $\mathrm{III}^{\mathrm{e}} \mathrm{s}$. apr. J.-C. L'arasement des vestiges dû à la réalisation de la piste de course en gore ne nous a pas permis de reconnaitre l'organisation des deux derniers états.

Enfin, le dernier sondage, également situé dans le talus, avait pour objectif de dégager à nouveau la partie septentrionale de la maison à la Mosaïque, fouillée en 1964. D'une superficie de $80 \mathrm{~m}^{2}$, il est situé à cheval entre les parcelles des « lits de briques » et de la maison à la Mosaïque. Une première domus augustéenne, dotée de sols en béton ou en terre battue, a été découverte. Aménagée directement sur le terrain naturel, son organisation nous échappe. Le riche mobilier (pied de cartibulum en schiste, verre millefiori...) récolté dans ses niveaux de démolition laisse supposer le statut élevé de ses occupants. Cette première domus est oblitérée par la construction de la maison à la Mosaïque au début du règne de Tibère. Un vaste vestibule, doté d'un sol en opus crustatum (marbres colorés), se développe sur un axe nord-sud et sépare une pièce d'habitat à l'est, d'une pièce d'habitat associée à un espace de service à l'ouest (cuisine ?). Cette domus est détruite suite à l'incendie qui ravage le quartier dans la première moitié $d u{ }^{\mathrm{e}}{ }^{\mathrm{e}} \mathrm{s}$. Suite à cet événement, cette parcelle n'est pas reconstruite et semble alors dévolue à un espace ouvert de type jardin, sans doute en lien avec la parcelle occidentale (dite " aux lits de briques »). Sur cette dernière, une pièce d'habitat (cubiculum ?) est construite au début de l'époque flavienne. Elle est dotée d'un plancher et décorée d'enduits peints $\mathrm{III}^{\mathrm{e}}$ style à candélabre (fig. 2). À l'instar du sondage $\mathrm{B}$, les niveaux d'incendie sont particulièrement bien conservés et permettent de repérer la présence d'un étage, également dévolu à de l'habitat (sol en béton). Les dernières phases de construction observées dans le sondage B ont pu être retrouvées, avec une séquence stratigraphique similaire. 
Fig. 2 - Emblema en opus sectile découvert dans l'atrium de la maison au Dauphin

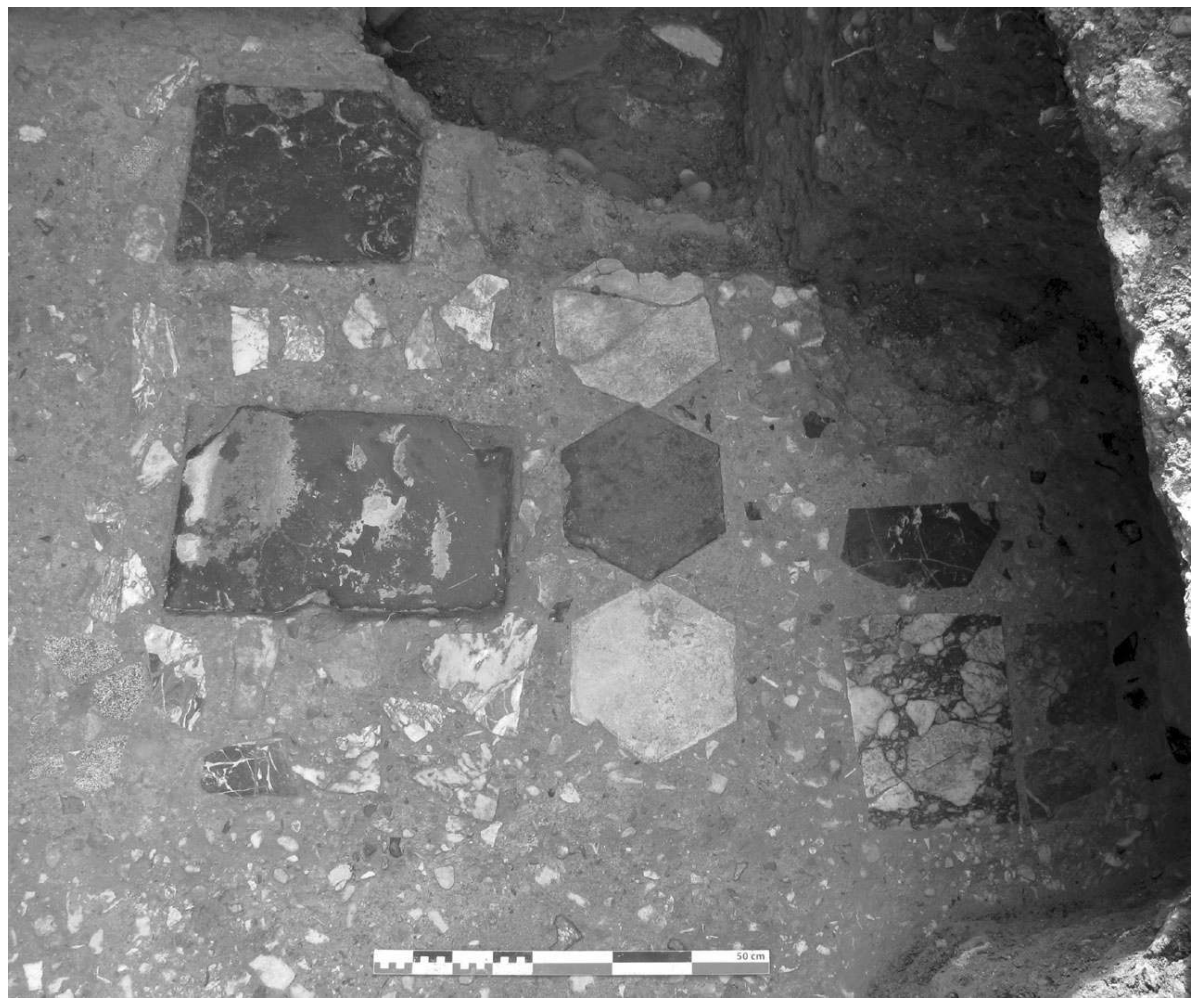

Sondage A.

Cliché : : B. Clément (université Lyon II). 
Fig. 3 - Peinture murale III style pompéien conservée en place (état IV)

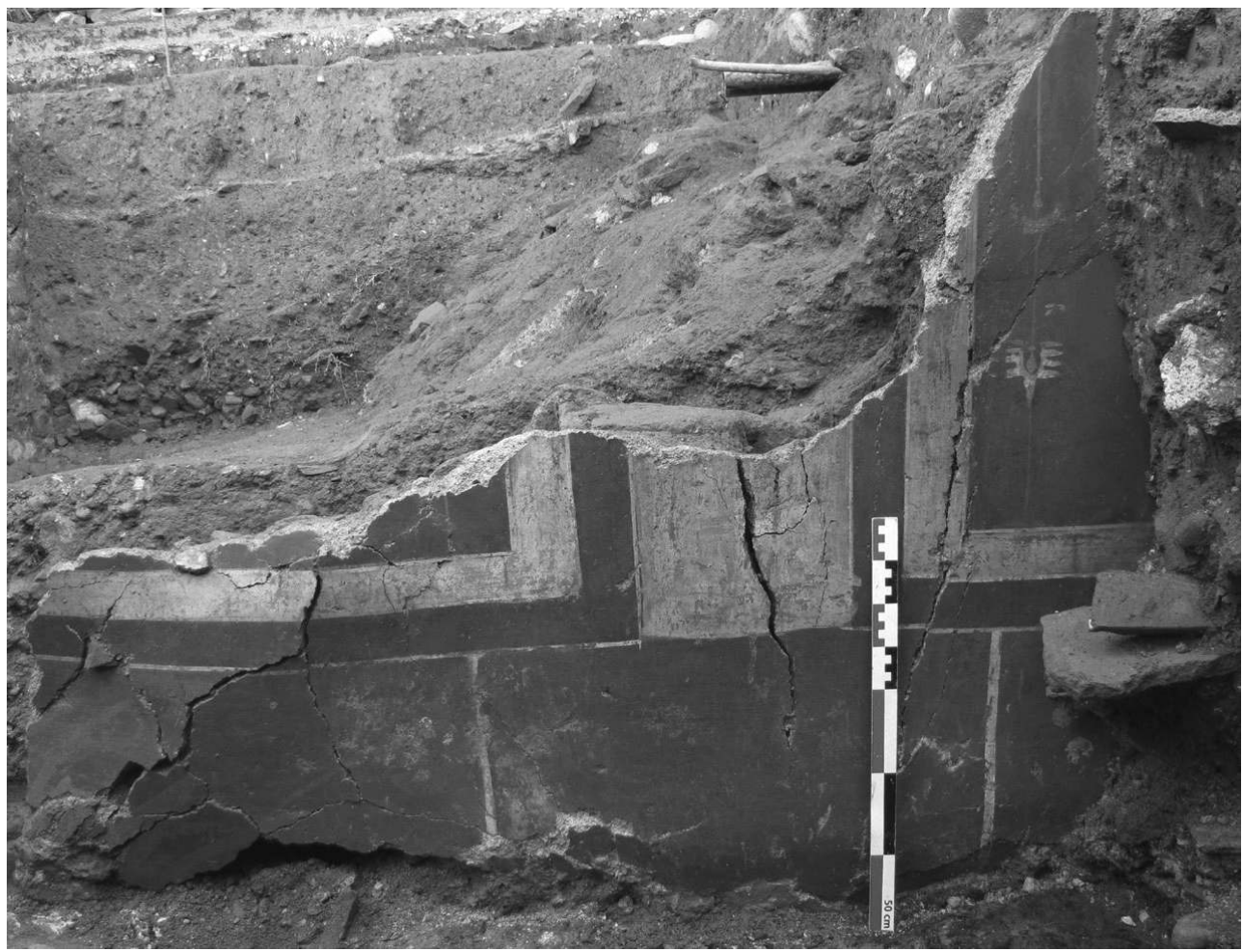

Sondage $\mathrm{C}$.

Cliché : B. Clément (université Lyon II).

\section{INDEX}

chronologie https://ark.frantiq.fr/ark:/26678/pcrtxT02uJOogm, https://ark.frantiq.fr/ark:/ 26678/pcrtof7EHNsS2e, https://ark.frantiq.fr/ark:/26678/pcrtZTmusVUU24 nature https://ark.frantiq.fr/ark:/26678/pcrtWWQS75V5Bc lieux https://ark.frantiq.fr/ark:/26678/pcrtSEeAipsBlD, https://ark.frantiq.fr/ark:/26678/ crtB8WDyqd6u9, https://ark.frantiq.fr/ark:/26678/pcrtdGSf7FYRvz, https://ark.frantiq.fr/ark:/ 26678/pcrtykDpsdYKiV

Année de l'opération : 2013

sujets https://ark.frantiq.fr/ark:/26678/pcrtKJVpuP3AET, https://ark.frantiq.fr/ark:/26678/ pcrtDlzbGxWvTo, https://ark.frantiq.fr/ark:/26678/pcrtbptj4SOA1W, https://ark.frantiq.fr/ark:/ 26678/pcrtaGBUR5Ekx1, https://ark.frantiq.fr/ark:/26678/pcrtBTWy6SwZAu, https:// ark.frantiq.fr/ark:/26678/pcrt20EsS32aXe, https://ark.frantiq.fr/ark:/26678/pcrtpDiX3ha6hS, https://ark.frantiq.fr/ark:/26678/pcrttrEq4Rcpkr, https://ark.frantiq.fr/ark:/26678/ pcrtRxLPuVsO8f, https://ark.frantiq.fr/ark:/26678/pcrtEaFZFvpInN, https://ark.frantiq.fr/ark:/ 26678/pcrtUt7XJjD0Si, https://ark.frantiq.fr/ark:/26678/pcrtRZ0ne3nzWk 
AUTEURS

BENJAMIN CLÉMENT

Université Lumière Lyon II 